\section{Commentary: Without missing a beat - Is preserving pulsatility the most important feature of the advanced ventricular assist device?}

\author{
David D. Yuh, MD, FACS, FACC
}

The advanced ventricular assist device (VAD) prototype Miyamoto and colleagues have developed ${ }^{1}$ addresses several important limitations with current VAD technologies, relevant to the broader clinical applications of longterm mechanical circulatory support. Of central interest is the novel sliding rotor mechanism of the advanced VAD that preserves arterial pulsatility with direct beat-tobeat dynamic coupling of pump flow with the native ventricles, optimizing diastolic preload and systolic afterload. Certainly, inducing pulsatility has been accomplished in other continuous-flow left ventricular assist devices through rotational speed modulation of the pump impeller ${ }^{2}$; however, this appears to be a less "physiologic" modality that would not appear to be as sensitive to dynamic changes in pre- and afterload conditions. That said, the clinical importance of maintaining arterial pulsatility remains uncertain and controversial. There is no lack of published evidence supporting the deleterious long-term physiologic consequences of diminished or absent pulsatility and greater shear-stress forces upon the peripheral microcirculation, ${ }^{3,4}$ including thrombogenesis (eg, diminished circulating von Willebrand factor), endothelial nitric oxide bioavailability, and end-organ function. However, clinically significant consequences of these largely theoretical manifestations have not been consistently observed in patients with endstage heart failure supported by continuous-flow devices

From the Department of Surgery, Stamford Hospital, Stamford, Conn.

Disclosures: The author reported no conflicts of interest.

The Journal policy requires editors and reviewers to disclose conflicts of interest and to decline handling or reviewing manuscripts for which they may have a conflict of interest. The editors and reviewers of this article have no conflicts of interest.

Received for publication July 1, 2020; revisions received July 1, 2020; accepted for publication July 2, 2020; available ahead of print July 26, 2020.

Address for reprints: David D. Yuh, MD, FACS, FACC, Department of Surgery, Stamford Hospital, One Hospital Plaza, P.O. Box 9317, Stamford, CT 06904 (E-mail:

DYuh@stamhealth.org).

JTCVS Open 2020;3:150-1

2666-2736

Copyright $(2020$ The Authors. Published by Elsevier Inc. on behalf of The American Association for Thoracic Surgery. This is an open access article under the CC BY-NCND license (http://creativecommons.org/licenses/by-nc-nd/4.0/).

https://doi.org/10.1016/j.xjon.2020.07.003

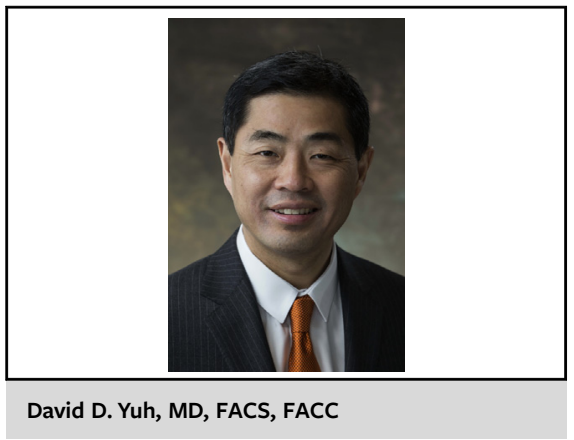

CENTRAL MESSAGE

The novel sliding rotor mechanism of the advanced ventricular assist device prototype shows promise in addressing several perennial limitations of current long-term circulatory assist devices.

nd certainly do not outweigh the markedly improved quality of life and prognoses conferred by these devices, including their pulsatile predecessors.

The intrinsic shut-off feature of the advanced VAD preventing regurgitant flow during pump stoppage is a valuable weaning feature of the sliding rotor mechanism, particularly since several centers have successfully weaned and explanted continuous-flow VADs in small-but-significant numbers of patients using different strategies to predict success. ${ }^{5,6}$ The ability to temporarily subtract VAD circulatory support may prompt more aggressive VAD-weaning trials. It is worth noting that cessation of pump flow for appreciable amounts of time during such weaning trials with this device may require special anticoagulation considerations, given the heightened risk of pump thrombosis. Regarding device durability, adding the sliding mechanism to the rotational action of the impeller might introduce an additional point of mechanical failure or vulnerability to thrombosis. For example, could fibrin deposition interfere with the sliding surfaces of the rotor within the pump housing, potentially leading to a fixed low-flow state?

Right ventricular failure following VAD implantation continues to be a vexing problem that is associated with significant perioperative mortality and morbidity. Although temporary right ventricular assist device (RVAD) support 
has been successfully used in instances of unexpected right ventricular failure, a significant number of these patients have failed weaning in several series and delayed RVAD implantation has been associated with high mortality rates. ${ }^{7}$ As a dedicated long-term RVAD has yet to be developed, the wider performance range of the advanced universal VAD presents a device more well suited to accommodate the lower-flow requirements and greater thrombosis risk of right-sided support.

Miyamoto and colleagues are to be congratulated for this in vivo "proof of concept" report of a novel adaptive advanced VAD mechanism that seeks to address some of the most persistent and challenging issues in long-term circulatory support.

\section{References}

1. Miyamoto T, Kado Y, Horvath DJ, Kuban BD, Sale S, Kukamachi K, et al. An advanced universal circulatory assist device for left and right ventricular support: first report of an acute in vivo implant. J Thorac Cardiovasc Surg Open. 2020;3: $140-8$.

2. Naito N, Nishimura T, Iizuka K, Takewa Y, Umeki A, Ando M, et al. Rotational speed modulation used with continuous-flow left ventricular assist device provides good pulsatility. Interact Cardiovasc Thorac Surg. 2018;26:119-23.

3. Vincent F, Rauch A, Loobuyck V, Robin E, Nix C, Vincentelli A, et al. Arterial pulsatility and circulating von Willebrand factor in patients on mechanical circulatory support. J Am Coll Cardiol. 2018;71: 2106-18.

4. Witman MA, Garten RS, Gifford JR, Groot HJ, Trinity JD, Stehlik J, et al Further peripheral vascular dysfunction in heart failure patients with a continuous-flow left ventricular assist device: the role of pulsatility. JACC Heart Fail. 2015;3:703-11.

5. Frazier $\mathrm{OH}$, Baldwin ACW, Demirozu ZT, Segura AM, Hernandez R Taegtmeyer $\mathrm{H}$, et al. Ventricular reconditioning and pump explantation in patients supported by continuous-flow left ventricular assist devices. J Heart Lung Transplant. 2015;34:766-72.

6. Knierim J, Heck R, Pieri M, Schoenrath F, Soltani S, Stawowy P, et al. Outcomes from a recovery protocol for patients with continuous-flow left ventricular assist devices. J Heart Lung Transplant. 2019;38:440-8.

7. Takeda K, Naka Y, Yang JA, Uriel N, Colombo PC, Jorde UP, et al. Outcome of unplanned right ventricular assist device support for severe right heart failure after implantable left ventricular assist device insertion. J Heart Lung Transplant. 2014; $33: 141-8$. 